\title{
Robust Fuzzy Neural Network Sliding Mode Control for Wind Turbine with a Permanent Magnet Synchronous Generator
}

\author{
K. Belamfedel Alaoui, S.Sefriti and I.Boumhidi \\ LESSI Laboratory, Department Of Physics, Faculty Of Sciences, Sidi Mohammed Ben Abdellah University, \\ Morocco \\ khawala.belamfedelalaouikhaoula@usmba.ac.ma; selma.sefriti@usmba.ac.ma; \\ iboumhidi@hotmail.com
}

\begin{abstract}
In the present paper, we are interested in the contribution of wind power to the electricity supply in power systems of small sized isolated communities. A robust fuzzy neural sliding control (FNSC) is proposed to track the maximum power point for an isolated wind energy conversion system using a permanent magnet synchronous generator (PMSG) with a hysteresis rectifier connected to a DC load. The turbine is controlled by a sliding mode controller (SMC) to reach the maximum power level. The main objective of the control is to adjust the rectifier voltage to provide it to the DC load as well as to maintain the maximum power extraction. In presence of large uncertainties and wind speed variations, the traditional SMC produces the chattering phenomenon due to the higher needed gain. In order to reduce this gain, FNSC is used for the estimation of the unknown part, thus provide lower gain. The stability of the proposed FNSC is analyzed by LYAPUNOV theory, simulations results are presented and the proposed control performance is shown by the comparison with the conventional SMC.
\end{abstract}

Keywords-variable speed wind turbine; permanent magnet synchronous generator; sliding mode control; fuzzy neural sliding mode control

\section{Introduction}

Wind energy is one of the most important renewable sources which are used in power systems either for stand alone or for grid connected applications. The use of wind energy to generate electricity is now accepted with a large industry manufacturing and installing thousands of Wind Turbines (WT) each year. Although, there are exciting new developments of wind turbines to make them more profitable [1]. Practically, there are two main types of vertical axis wind turbines: fixed speed and variable speed [2]. In this study, we consider the case of variable speed, due to its great ability in the extraction of energy [3].This work is devoted to the electrical part with the aim of designing a robust controller to adjust the rectifier voltage, to keep at the same time the captured energy at its maximum and to reduce the chattering phenomenon due to the higher needed gain. . The output tracking of non-linear WT system with uncertainties using conventional Proportional Integral Derivative (PID) controller is quite complex, since conventional PID controllers are tuned to a particular operating point. This degrades the performance of PID controller under non-linearities. Developing a simple and robust controller for uncertain non-linear WT system is important to overcome this problem. 
K. Belamfedel Alaoui, S. Sefriti and I.Boumhidi, Robust Fuzzy Neural Network Sliding Mode Control for Wind

Turbine with a Permanent Magnet Synchronous Generator. Transactions on Machine Learning and Artificial Intelligence, Vol 5 No 4 August (2017); p: 725-735

Sliding mode control ([4]-[6]), emerges as an especially suitable option to deal with variable speed wind turbine. This is due to its robustness with uncertainties and disturbances. Therefore, when the uncertainties are large, a high discontinuous control gain is needed and higher amplitude of chattering is produced.

To control nonlinear uncertain dynamic systems, adaptive fuzzy neural control has been used to solve approximation functions problems. The fuzzy neural network (FNN) has proved in many applications that it possesses the propriety of universal approximation [7].

The designed method is a combination of conventional SMC and FNN with online adaptation of the parameters to adjust the stator voltages of the PMSG. The proposed control combines the advantages of the FNN and those of SMC because of its ability to predict unknown part in the equivalent control term, so the gain used in the robust control term becomes smaller which helps the network error to be compensated.

This study is organized as follows. The next section presents the system modeling and control objectives. Section 3 shows the design of the proposed control strategies for power capture optimization. In Section 4 , simulation results are provided to demonstrate the robust control performance of the proposed approach. Finally, a concluding remark is given in section 5 .

\section{System Modeling and Control Objectives}

The proposed system contains a variable speed wind turbine coupled directly to a PMSG, a rectifier and a DC load as it is illustrated in figure 1.

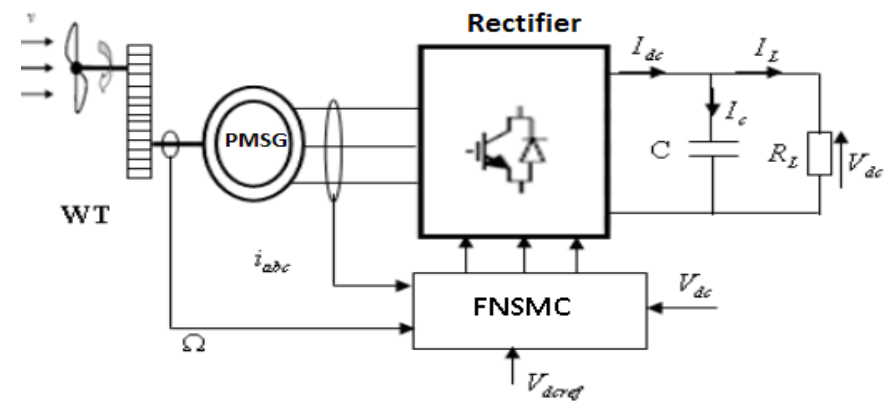

Figure 1: The structure of the studied system

\subsection{Wind Turbine Modeling}

Wind energy across a surface $S$ depends on the cube of the wind speed $v$ and the density of the air $\rho$ .This energy is given by:

$$
P_{v}=\frac{1}{2} S \rho v^{3}
$$

We are interested in a vertical axis turbine where the active surface is in function of the geometric dimensions of the wing shown in figure 2:

$$
S=2 \cdot R \cdot H
$$




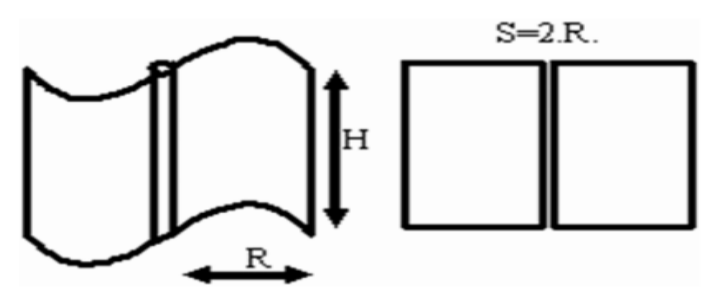

Figure 2: Geometric dimensioning Savonius wing

$R$ is the radius of the rotor and $H$ is the height of the turbine

The aerodynamic power captured by the rotor is given as follows:

$$
P_{a}=\frac{1}{2} \rho \pi R^{2} C_{p}(\lambda, \beta) v^{3}
$$

The power coefficient $C_{p}$ depends on the blade pitch angle $\beta$ and the tip-speed ratio $\lambda$ which is defined as follows:

$$
\lambda=\frac{\omega_{t} R}{v}
$$

With $\omega_{t}$ is the rotor speed.

The power coefficient $C_{p}(\lambda, \beta)$ is a nonlinear function of $\lambda$ and $\beta$ [8]:

$$
C_{p}(\lambda, \beta)=C_{1}\left(\frac{C_{2}}{\lambda_{i}}-C_{3} \beta-C_{4}\right) \exp \left(-\frac{C_{5}}{\lambda_{i}}\right)+C_{6} \lambda
$$

Where:

$$
\frac{1}{\lambda_{i}}=\frac{1}{\lambda+0.08 \beta}-\frac{0.035}{\beta^{3}+1}
$$

And the parameters $c_{i}$ are known constants without units.

In this paper, we focus on the modeling of the electrical part of the variable speed wind turbine, which is presented by PMSG.

The equations of PMSG in a d-q rotating frame linked to the rotor as follows [9]:

$$
\left\{\begin{array}{l}
V_{s d}=R_{s} I_{s d}+L_{s d} \frac{d}{d t} I_{s d}-p w L_{s q} I_{s q} \\
V_{s q}=R_{s} I_{s q}+L_{s q} \frac{d}{d t} I_{s q}+p w L{ }_{s d} I_{s d}+p w \phi_{f}
\end{array}\right.
$$

With:

$R:$ is the resistance of the stator windings,

$I_{s d} I_{s q}$ : are the stator currents in the Park rotating frame, $V_{s d} V_{s q}$ : are the stator voltage in the Park rotating frame, $L_{s d} L_{s d}$ : are Inductances along the direct and the quadrature axes, $p:$ is the number of pole pairs, 
$\omega=p . \Omega$ : Voltage pulsation $(\mathrm{rad} / \mathrm{s})$,

And $\phi_{f}$ is the magnetic flux created by the PMSG through the stator windings.

The electromagnetic torque in the rotating frame is given by:

$$
T_{e}=\frac{3}{2} p\left[\left(L_{s d}-L_{s q}\right) I_{s d} I_{s q}-\phi{ }_{f} I_{s q}\right]
$$

\subsection{Control Objectives}

Below the nominal power, the main control objective is to define a resistive electromagnetic torque $T_{e}$ to be provided by the generator in order to optimize the energy captured from the wind. The power coefficient curve $C_{p}(\lambda, \beta)$ has a unique maximum which corresponds to the optimal wind energy (see Figure 3):

$$
C_{p}\left(\lambda_{\text {opt }}, \beta_{\text {opt }}\right)=C_{\text {popt }}
$$

The rotor, thus, provides maximum aerodynamic power only to the tip-speed $\lambda_{\text {opt }}$ :

$$
\lambda_{\text {opt }}=\frac{\omega_{\text {topt }} R}{v}
$$

To maximize the captured energy of the wind, the variables $\lambda$ and $\beta$ must be maintained at their optimal values in order to ensure maximum value of $C_{p}$. So, the blade pitch angle is fixed at its optimal value $\beta_{\text {opt }}$ . The tip-speed $\lambda$ depends on both of the wind speed $v$ and the rotor speed $\omega_{t}$. As the wind speed is not a controllable input, the rotor speed $\omega_{t}$ must be adjusted by $T_{e}$, to track the optimal reference given by:

$$
\omega_{\text {topt }}=\frac{\lambda_{\text {opt }}}{R} v
$$

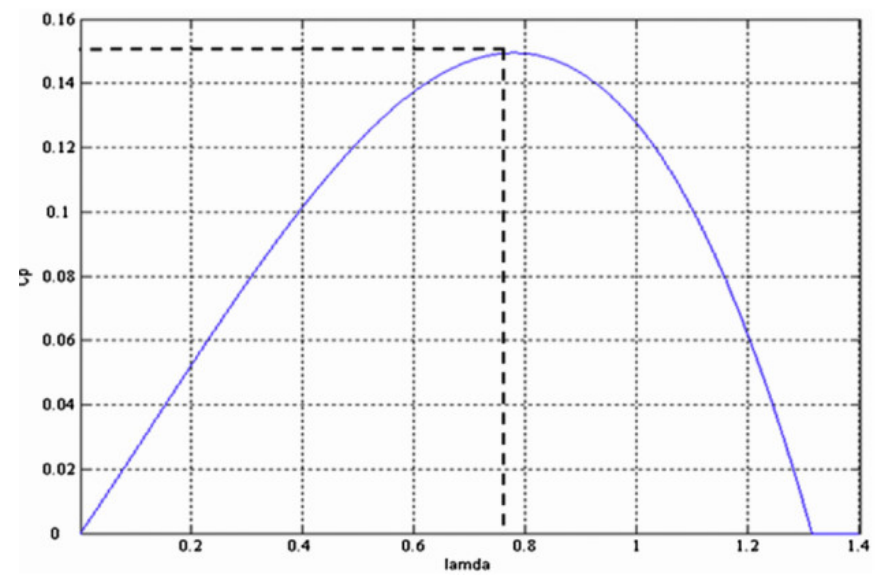

Figure 3: Power versus tip speed ratio for $\beta=\beta_{o p t}$ 


\section{Fuzzy Neural Network Sliding Mode Control strategy}

\subsubsection{Conventional Sliding Mode Controller (SMC)}

SMC is one of the most important robust control approaches with respect to system dynamics and invariant to uncertainties. LYAPUNOV stability approach is used in SMC to keep the nonlinear system under control. Our target is to control the stator current of the PMSG and the DC voltage even with the wind speed variations.

Let define the control vector $u=\left[V_{s d} V_{s q}\right]=\left[u_{1} u_{2}\right]$ and the output vector $y=\left[V_{d c} I_{d}\right]$ and the system state $x=\left[I_{s d} I_{s q}\right]^{T}$

The model described by (7) can be rewritten in the state space as follows:

$$
\begin{aligned}
& \left\{\begin{array}{l}
\dot{X}=f(x)+G(x) U+\xi \\
y=\left[y_{1} y_{2}\right]
\end{array}\right. \\
& \dot{X}=\left[\begin{array}{l}
a_{11} x_{1}+a_{12} x_{2} \\
a_{21} x_{1}+a_{22} x_{2}+a_{23}
\end{array}\right]+\left[\begin{array}{ll}
g_{1} & 0 \\
0 & g_{2}
\end{array}\right] U+\xi
\end{aligned}
$$

With

$$
\begin{aligned}
& a_{11}=\frac{R_{s}}{L_{s d}}, a_{12}=\omega \frac{L_{s q}}{L_{s d}}, a_{21}=\frac{R_{s}}{L_{s q}}, a_{22}=\omega \frac{L_{s d}}{L_{s q}} \\
& a_{23}=\omega \frac{\phi_{f}}{L_{s q}}, g_{1}=\frac{1}{L_{s d}}, g_{2}=\frac{1}{L_{s q}} \text { and } \xi \text { the unknown term. }
\end{aligned}
$$

In the following, we have two tracking errors since we have two control laws. The tracking errors of the system are defined as:

$$
\begin{aligned}
& e_{1}(t)=I_{s d}(t)-I_{s d} r e f(t) \\
& e_{2}(t)=I_{s q}(t)-I_{s q} r e f(t)
\end{aligned}
$$

To design the SMC there are two steps. First finding the sliding surface second, developing the control law.

Generally, the sliding surface is defined as:

$$
\sigma(t)=\left(\gamma+\frac{d}{d t}\right)^{r-1} e(t)
$$

where $r$ is the order of the system and $\gamma$ is a positive constant.

The relative degree of the system (12) is 1 . Then, the sliding surfaces can be defined as:

$$
\begin{gathered}
\sigma_{1}=e_{1} \\
\sigma_{2}=e_{2}
\end{gathered}
$$

Differentiating $\sigma$ with respect to time, we have: 


$$
\begin{gathered}
\dot{\sigma}_{1}=f_{1}(x)+g_{1} u_{1}-\dot{I}_{s d}-r e f+\xi \\
\dot{\sigma}_{2}=f_{2}(x)+g_{2} u_{2}-\dot{I}_{s q}-r e f+\xi
\end{gathered}
$$

To guarantee the existence of sliding mode on a switching surface in finite time, the condition given below has to be satisfied [5]:

$$
\sigma \sigma^{\circ}<0
$$

The control law that satisfies Eq. (20) is given by [9]:

$$
\begin{aligned}
& u_{1}=-\frac{f_{1}(x)-\dot{I}_{s d}-r e f}{g_{1}}-\frac{k_{1}}{g_{1}} \operatorname{sign}\left(\sigma_{2}\right) \\
& u_{2}=-\frac{f_{2}(x)-\dot{I}_{s q}-r e f}{g_{2}}-\frac{k_{2}}{g_{2}} \operatorname{sign}\left(\sigma_{2}\right)
\end{aligned}
$$

Where $k_{1}$ and $k_{2}$ are the positive switching gains to compensate the uncertainties. With $\operatorname{sign}($.$) is the sign$ function, given by:

$$
\operatorname{sign}(\sigma)= \begin{cases}1 & \text { if } \sigma>0 \\ 0 & \text { if } \sigma=0 \\ -1 & \text { if } \sigma<0\end{cases}
$$

To eliminate the chattering effect caused by the discontinuous control law, the boundary layer approach can be used. The control becomes as follows:

$$
u_{1,2}=-\frac{f_{1,2}(x)-\dot{I}_{d, q}-r e f}{g_{1,2}}-\frac{k_{1,2}}{g_{1,2}} \text { sat }\left(\sigma_{1,2}\right)
$$

Where sat (.) is the saturation function, given by:

$$
\operatorname{sat}(\sigma)= \begin{cases}\sigma / L & \text { if }|\sigma|<L \\ \operatorname{sign}(\sigma) & \text { otherwise }\end{cases}
$$

With $L$ is the boundary layer thickness.

\subsection{Fuzzy Neural Network Representation}

The fuzzy neural network considered is formed by five layers. The first one is for the inputs; the second one is formed by the memberships, the third one contains the rules; the fourth one is the normalized and the output layer receiving the weighted outputs. The weights are adjustable to estimate the unknown term $\xi$.

This network is illustrated in figure 4. 


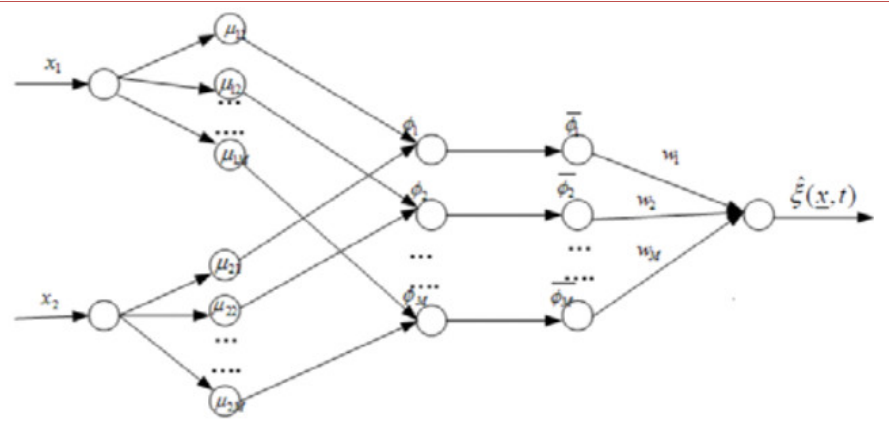

Figure 4 The proposed structure of the fuzzy neural network

We describe in the following the function of each layer:

Layer1: it's the input layer which contains the input variables $x_{1,2}$, transmits directly input values to the next layer.

Layer2: in this layer, each node performs a membership function and acts as a unit of memory. The Gaussian function is adopted as the membership function. For the $k^{\text {th }}$ input, the corresponding net input and output of the $\mathrm{I}^{\text {th }}$ node can be expressed as:

$$
\mu_{k}^{l}\left(x_{k}\right)=\exp \left(-\frac{\left(x_{k}-c_{k}^{l}\right)^{2}}{\left(\sigma_{k}^{l}\right)^{2}}\right)
$$

Where $c_{k}^{l}$ and $\sigma_{k}^{l} ; l=1,2, \ldots, M$ are the mean and standard deviation of the Gaussian function of the $I^{\text {th }}$ partition for the $k^{\text {th }}$ input variable $x_{k}$, respectively, and $M$ is the total number of fuzzy rules.

Layer3: this layer represents one fuzzy logic rule and performs prediction matching of a rule. The output of a rule node in this layer is calculated by the product operation as follows:

$$
\phi(x)=\prod_{k=1}^{2} \mu_{k}^{l}\left(x_{k}\right)
$$

Layer4: in this layer, each of these firing strengths of the rules is compared with the sum of all the firing strengths. Therefore, the normalized firing strengths are computed in this layer as,

$$
\bar{\phi}(x)=\frac{\phi(x)}{\sum_{l=1}^{M} \phi(x)}
$$

Layer5: this is the output layer calculates the summation of its input values from the previous.

$$
\hat{\xi}(x)=\sum_{l=1}^{M} w_{l} \phi \overline{(x)}
$$

The FNN prediction error is $w$ given in the next section. 
The aim of the learning is to adjust the weights of, $w_{S}^{l}, \sigma_{S}^{l}$ and $c_{S}^{l}$. The online Learning algorithm is a gradient descent (GD) search algorithm in the space of the network parameters. The essence of (GD) consists of iteratively adjusting the weights the direction opposite to the gradient of error, so as to reduce the discrepancy according to:

$$
\dot{w}_{l}=\eta_{w l} w \phi
$$

Where $\eta_{w l}$ is the learning rate for $w_{y}^{l}$.

The updated laws of $c_{\varsigma}^{l}$ and $\sigma_{\varsigma}^{l}$ also can be obtained by gradient descent search algorithm:

$$
\dot{c}_{k}^{l}=2 \eta_{c_{k}^{\prime}} w \phi w_{l} \frac{\left(x_{k}-c_{k}^{l}\right)}{\left(\sigma c_{k}^{l}\right)^{2}}
$$

And

$$
\sigma_{k}^{l}=2 \eta_{\sigma_{k}^{l}}^{l w \phi w} \frac{\left(x_{k}-c_{k}^{l}\right)^{2}}{\left(\sigma_{k}^{l}\right)^{3}}
$$

Where $\eta_{\mathcal{C}_{k}}$ and $\eta_{\sigma_{k}^{\prime}}$ are the learning-rate parameters of the mean and the standard deviation of the Gaussian functions.

\subsection{Proposed Fuzzy Neural Sliding Controller (FNSC)}

We are interested in implementation of a robust control of nonlinear system, uncertain and disrupted as (12).

To develop the control law, we propose to approximate the unknown functions $\xi$ by the FNN presented in figure 4.

Let denote the approximation error as: $w=\xi-\hat{\xi}$

With $|w|<w^{*}$ and $w^{*}$ is the upper bound of the network error supposed known.

Theorem: Consider the system described by (12) in the presence of uncertainties. If the system control is designed as:

$$
U_{1,2}=g_{1,2}-1\left(-f_{1,2}(x)-\hat{\xi}+\dot{I}_{d, q}-\operatorname{ref}-\operatorname{Ksat}\left(\sigma_{1,2}\right)\right)
$$

Where $w^{*}+\eta<k$; with $k$ is a small positive vector values

The trajectory tracking error will converge to zero in finite time.

Proof: we consider the LYAPUNOV function:

$$
V=\frac{1}{2} \sigma^{2} \quad \text { Then } \dot{V}=\sigma \dot{\sigma}
$$




$$
\dot{V}=\sigma\left(f_{1,2}(x)+g_{1,2}(x) u_{1,2}-\dot{I}_{d, q-r e f}+\xi-\text { Ksat }(\sigma)\right)
$$

By replacing the expression of $u$ given in the theorem we have:

$$
\begin{aligned}
& \dot{V}=\sigma(\xi-\hat{\xi}-k s a t(\sigma)) \\
& =\sigma(w-k s a t \quad(\sigma)) \\
& =\sigma w-k \sigma \text { sat }(\sigma) \\
& \leq|\sigma \| w|-k \sigma s a t \quad(\sigma)<|\sigma| w-k \sigma s a t \quad(\sigma)
\end{aligned}
$$

By choosing $w^{*}+\eta<k$ we have:

For any $L>0$, if $|\sigma|>L, \operatorname{sat}(\sigma)=\operatorname{sign}(\sigma)$ the function $\dot{V}=\left(w^{*}-k\right)|\sigma|<0$. However, in a small L-vicinity of the origin [5], (boundary layer), sat $(\sigma)=\frac{\sigma}{L}$ is continuous, the system trajectories are confined to a boundary layer of sliding mode manifold $\sigma=0$.

\section{Simulation Results}

In order to make a comparison between the proposed control strategies, different simulations are considered for the case of the PMSG with:

A wind speed profile of $10 \mathrm{~m} / \mathrm{s}$ means value.

$$
\begin{aligned}
& C_{1}=0.5176, C_{2}=116, C_{3}=0.4, C_{4}=5, C_{5}=21, C_{6}=0.0068 \\
& R=0.5 m, \rho=1.29 \mathrm{~kg} / \mathrm{m}^{3}, H=2 m S=2 m^{2} \\
& L_{s}=0.0263 \mathrm{H}, R_{s}=1.4 \Omega C_{p \max }=0.15 \\
& \phi_{f}=0.15 \mathrm{~Wb}, R_{L}=80 \Omega, C=500 \mu \mathrm{F}
\end{aligned}
$$

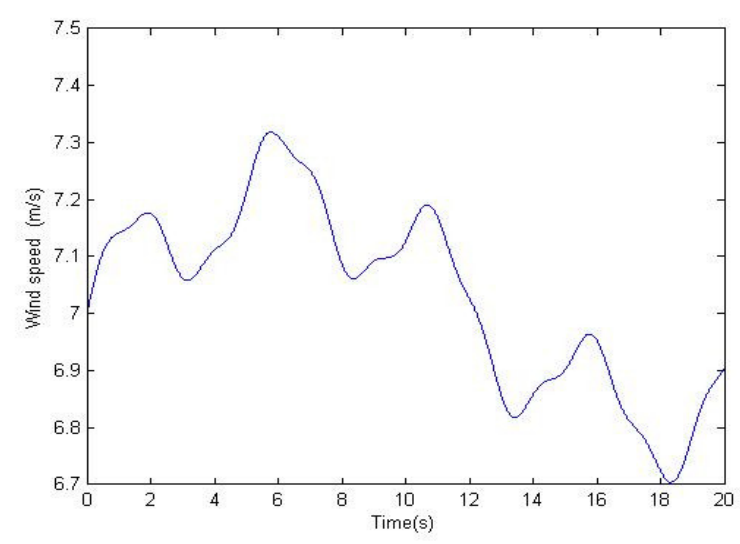

Figure 5 Wind speed profile $(\mathrm{m} / \mathrm{s})$ 


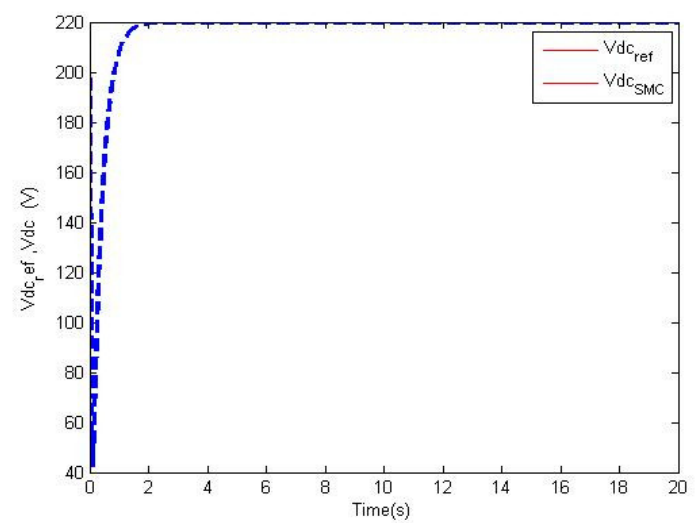

Figure 6 SMC DC voltage and reference voltage without uncertainties

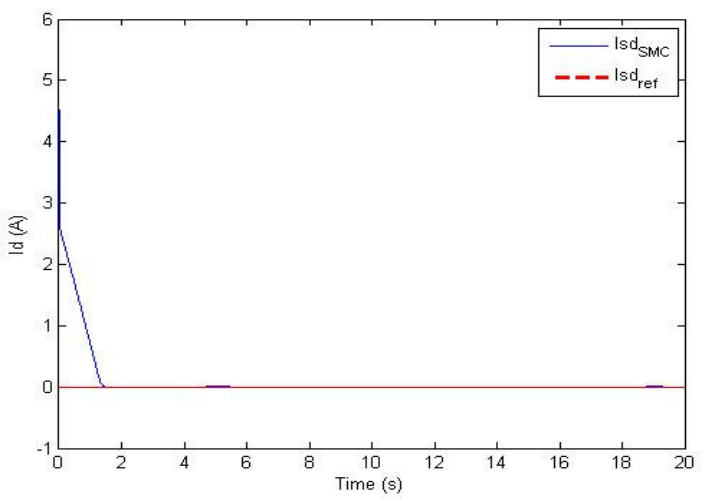

Figure 7 Direct current by traditional sliding mode control without uncertainties

Three membership functions have been used for each of the two inputs $\left(I_{s d} I_{s q}\right)$ of the FNN.

Let now consider the uncertainties as a vector random noise with the magnitude equal.

$$
\xi=10+0.3 \times \operatorname{rand}()
$$

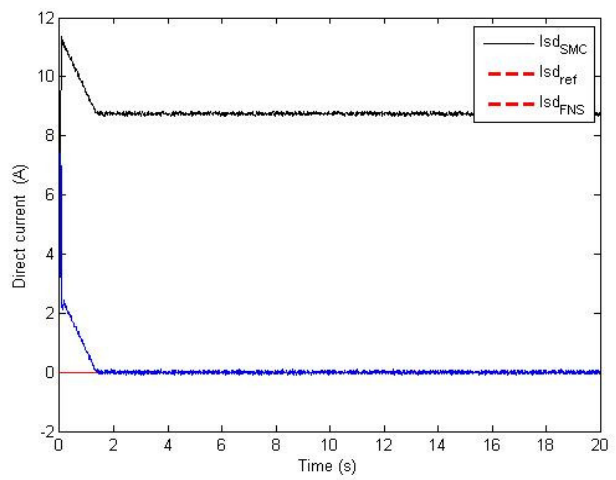

Figure 8 Direct current by the fuzzy neural sliding control and SMC

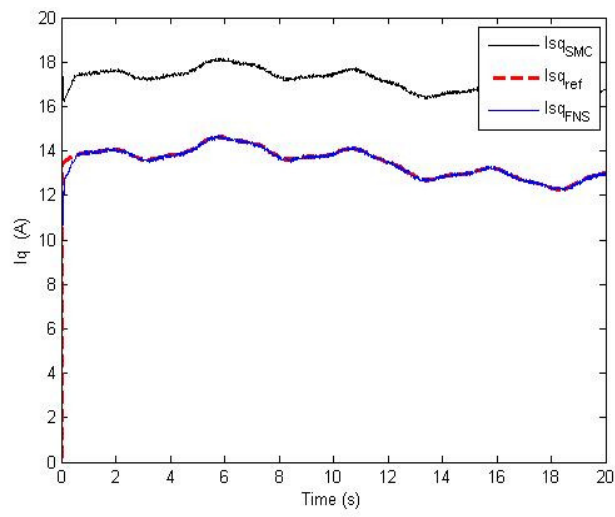

Figure 9 stator current by the fuzzy neural sliding control and SMC

Figure6 presents the response of the DC voltage by the proposed control and the desired one; Figure7 presents the direct current controlled by traditional sliding mode control without any disturbances; Figure8 and Figure9 present a comparison between the traditional sliding mode control and the proposed fuzzy neural sliding mode control in the presence of disturbances. From these figures, it can be seen that the best performance is obtained when the proposed approach is applied.

\section{Conclusion}

In this study, a control of a permanent magnet synchronous generator and the DC voltage feeding a hysteresis rectifier is presented. The proposed control strategy is based on nonlinear predictive fuzzy neural sliding control. The comparison with the traditional sliding mode control has been realized and 
simulation results have shown a good performance of the proposed method to track the optimal reference without any oscillatory behavior. The proposed model has been implemented using Matlab.

\section{REFERENCES}

[1] J. F. Manwell, J. G. McGowan and A.L. Rogers, Wind Energy Explained: Theory, Design and Application, New York: John Wiley \& Sons,2002.

[2] G. Ofualagba and E.U. Ubeku, “Wind energy conversion system-wind turbine modelling," IEEE Power and Energy Society General Meeting - Conversion and Delivery of Electrical Energy in the 21st Century, pp. 1$8,2008$.

[3] T. Burton, D.Sharpe,N. Jenkins and E. Bossanyi, Wind Energie Handbook. New York: John Wiley \& Sons, 2001.

[4] V. I. Utkin, Sliding Modes in Control Optimization, Berlin: Springer-Verlag, 1992.

[5] J. J. Slotine, "Sliding controller design for non-linear systems," International Journal of Control, vol. 40, pp. 421-434, 1984.

[6] V. Utkin, J. Guldner and J. Shi, Sliding Mode Control in Electromechanical System, London: Taylor \& Francis, 1999.

[7] E-M. Boufounas, J. Boumhidi and I. Boumhidi, "Optimal $\mathrm{H} \infty$ control without reaching phase for a variable speed wind turbine based on fuzzy neural network and APSO algorithm", International Journal of Modelling, Identification and Control, Vol. 24, No. 2, pp. 100-109, 2015.

[8] H.P. Wang, A. Pintea, N. Christov, P. Borne and D. Popescu, "Modelling and recursive power control of horizontal variable speed wind turbines," Journal of Control Engineering and Applied Informatics, vol. 14, no. 4, pp. 33-41, 2012.

[9] D .Cardenas, "Control of wind turbine using a switched reluance generator". Phd thesis university of Nottingham.1996.

[10] E-M. Boufounas, J. Boumhidi and I. Boumhidi, “Optimal neural network sliding mode control for a variable speed wind turbine based on APSO algorithm", Digests $2^{\text {nd }}$ Annual Conf. IEEE on Complex Systems (WCCS'14) Morocco, p. 419-424, 2014. 